\title{
Isolation and Initial Study of Ø1M3-16, a Bacteriophage Infecting an Alkaliphilic Vibrio metschnikovii Isolate from Lake Magadi, a Soda Lake in Kenya's Great Rift Valley
}

\author{
K. D. Moulton*, M. Movassaghi*, J. L. Jamison*, N. Lobo*, C. Lobo*, W. Witbeck*, K. Gikonyo*, \\ J. Gaynor*, L. J. Rothschild**, F. Mwaura***, and S. M. Duboise* \\ * University of Southern Maine, Department of Applied Medical Sciences, 96 Falmouth Street, 178 \\ Science Bldg., Portland, ME 04103 \\ ** NASA Ames Research Center, Mail Stop 239-20, Moffett Field, CA 94035 \\ *** University of Nairobi, Department of Geography and Environmental Studies, University Way, \\ P.O. Box 30197, GPO100, Nairobi, Kenya
}

Bacteriophages are critically important in the evolution, population fluctuations, and ecologically complex disease associations of bacteria of the family Vibrionaceae [1]. Bacteriophages are not only the most abundant biological entities on Earth, they also have powerful impact in manipulating the biology and evolution of their hosts in remarkable ways [2]. Investigation of vibriophages has been particularly important and extensive in relation to understanding the biology of Vibrio cholerae and the pathogenesis and epidemiology of cholera [3]. Thus our isolation of an alkaliphilic bacterium identified as Vibrio metschnikovii from a saline and alkaline warm spring environment at Lake Magadi, a soda lake in Kenya's Great Rift Valley, has been followed by effort to isolate vibriophages that infect this microbe as we study the microbial and viral biodiversity of this extremely saline and alkaline environment. Here we report isolation of a soda lake lytic vibriophage that infects this alkaliphilic Vibrio metschnikovii isolate.

Microbes of Lake Magadi in Kenya's Great Rift Valley are capable of living in hypersaline and alkaline habitats of $\mathrm{pH} 11$ and higher that are formed through deposition of sodium sesquicarbonate by thermal springs combined with high rates of evaporation in a hot and arid climate. Isolation of an organism identified as Vibrio metschnikovii from Kenyan soda lakes has also been reported by others [4]. Recently, we have used our isolate to enrich for bacteriophages specific for this host that can be detected in the waters near the warm spring where the microbe was isolated. This soda lake Vibrio metschnikovii isolate is cultivated in alkalophile medium described previously [5] using broth or solid agar-based formulations as appropriate. The lytic bacteriophage Ø1M3-16 was enriched in culture when a logarithmically growing culture of Vibrio metschnikovii was mixed with the Lake Magadi warm spring water and incubated at ambient temperature overnight and then stored at $4^{\circ} \mathrm{C}$ awaiting further processing. After diluted samples of the enrichment culture were mixed with a $0.5 \%$ soft agar overlay of the medium with the host organism in petri dish cultures, viral plaques were observed and picked for further virus isolation and propagation as a clonal population

Ultrastructure of the bacteriophage $Ø 1 \mathrm{M} 3-16$ was observed in negatively stained preparations for transmission electron microscopy using the Tecnai BioTwin TEM at the University of Southern Maine and is shown in Figure 1. As with other bacteriophages we have isolated at Lake Magadi and previously reported [6], the bacteriophage $\varnothing 1 \mathrm{M} 3-16$ is a tailed phage that can be classified based on morphology as belonging to the Order Caudovirales. The viral morphotype observed is most consistent with this phage being a member of the virus family Siphoviridae. Genomic DNA of bacteriophage Ø1M3-16 was isolated using a Lambda DNA isolation kit from Qiagen Inc. 
(Valencia, CA) and analyzed initially by restriction endonuclease digestion which indicated a probable genome size of less than 30 kilobases of DNA. Genomic sequencing and annotation was begun using plasmid libraries constructed by a project-based graduate level molecular biology laboratory class at the University of Southern Maine which produced phage DNA restriction fragment libraries in pNEB193 from New England BioLabs (Ipswich, MA). Completion of genomic analysis has proceeded using a genomic library constructed in the pSMART vector (Lucigen, Inc., Middleton, WI) along with primer walking to fill in any sequence gaps. The annotated Ø1M3-16 genomic sequence and genomic organization is being compared with known bacteriophage sequences [7].

\section{References}

[1] M.K. Waldor et al., Phages: Their Role in Bacterial Pathogenesis and Biotechnology. ASM Press, Washington, D.C., 2005.

[2] F. Rohwer and R.V. Thurber, Nature 459 (2009) 207.

[3] E.J. Nelson et al., Nature Reviews: Microbiology 7 (2009) 693.

[4] R. Mwirichia et al., Extremophiles 14 (2010) 339.

[5] R.M. Atlas, Handbook of Microbiological Media (Third Ed.), CRC Press, Boca Raton, FL

[6] J.L. Jamison et al., Microsc. Microanal. 16 (Suppl. 2) (2010) 1962.

[7 ] This research was supported by National Science Foundation grant DGE-0440560, international supplement (DGE-0749059), and the Maine Space Grant Consortium NASA EPSCoR RID Grants EP-07-03 and EP-08-02. TEM capabilities at USM were established with combined NSF (CNS-0521262) and USM support.

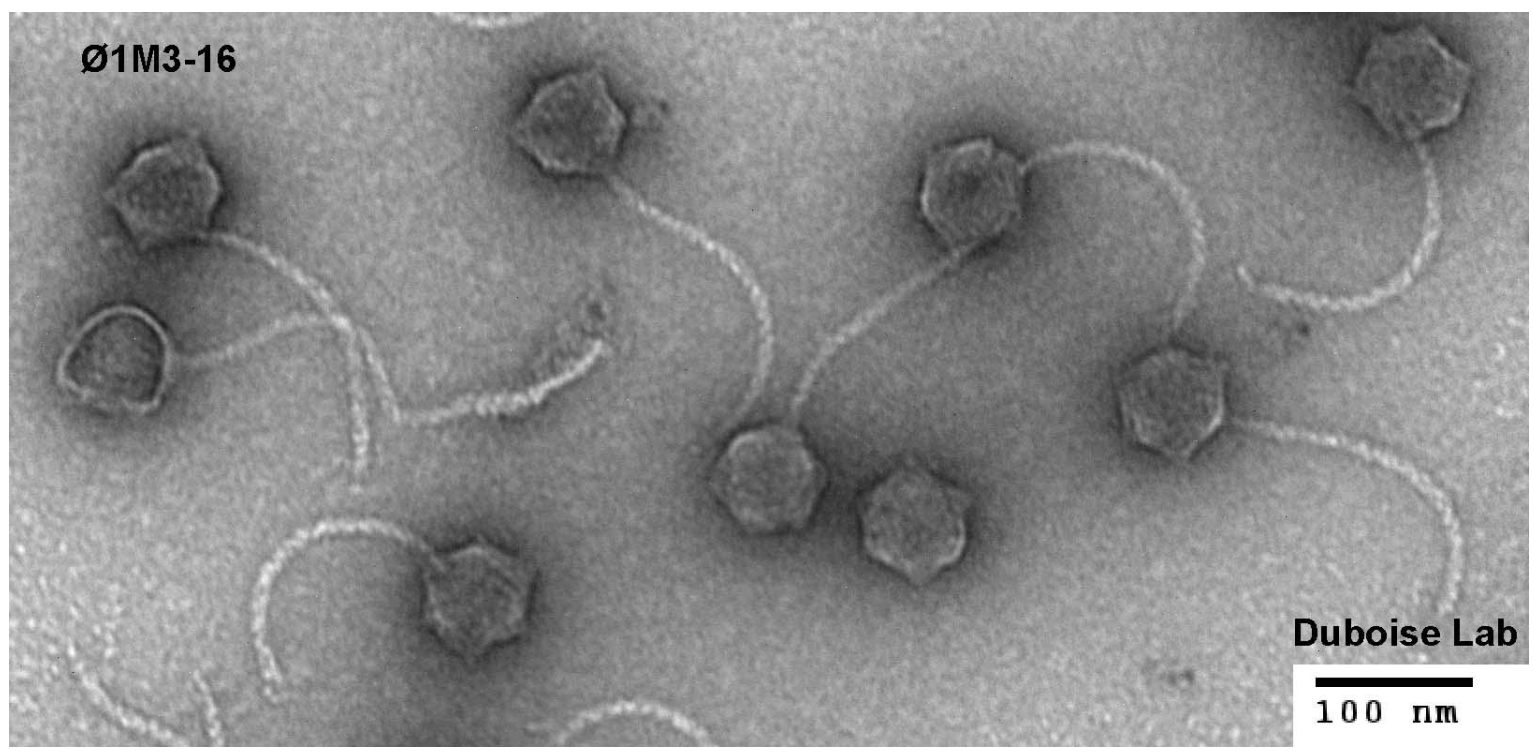

Figure 1: 1\% UA stained TEM images of bacteriophage Ø1M3-16 that infects a Lake Magadi isolate of Vibrio metschnikovii are shown. This tailed bacteriophage has a morphotype consistent with the family Siphoviridae. Scale bar $=100 \mathrm{~nm}$. 